\title{
Hernán Nottoli $\mid$ Methods for Evaluation in Mathematics for Architecture and Design
}

In mathematical teaching, there exists a dichotomy between two entities. On the one hand, there are the methodologies for imparting mathematical knowledge; on the other hand there are different mechanisms for evaluating students. Hernán Nottoli analyses some aspects that we consider relevant with respect to how knowledge is verified and ranked in evaluation tests in the case of mathematics in architecture and design schools, provides statistical evidence of experiences in the Faculty of Architecture, Urbanism and Design at the University of Buenos Aires, and provides an example of the kind of exercise that has been used with success with his own students

\section{Introduction}

In mathematical teaching, there exists a dichotomy between two entities. On the one hand, there are the methodologies for imparting mathematical knowledge; on the other hand there are different mechanisms for evaluating students. Unfortunately, both are normally isolated areassomething which should not have to operate in those terms-and in addition, that divorce is repeated in all levels of introductory courses.

In this paper we will analyse some aspects that we consider relevant with respect to how knowledge is verified and ranked in evaluation tests.

Although the mechanisms are analogous in other disciplines, this paper fundamentally analyses the case of mathematics in architecture and design schools. One particular characteristic of this area is that students in the design disciplines have a scarce affinity for the knowledge of the field of mathematics.

Finally we will suggest some proposals (see Example 1) based on the steps of methodology set forth that could optimise the objectivity of the information being evaluated, as well as the results obtained, to impart knowledge to the students and to make possible the subsequent transfer of that knowledge to other disciplines.

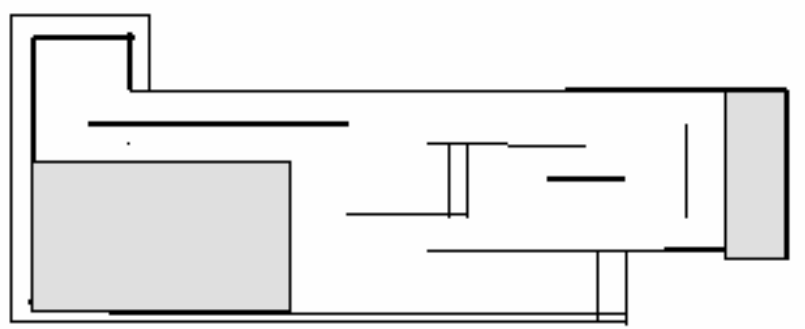

Example 1: Try to find an Eulerian path in order to pass through every space in the Barcelona Pavillion of Mies Van der Rohe, 1929 (see the solution at the end of this paper) 


\section{Development of the theme}

With regards to teaching mathematics, there are usually absolute differences between the methods used to impart knowledge and in the mechanism for evaluating the students.

The logical reasoning of mathematics is fundamentally based on the process called deduction. Let us focus on the classical case, mentioned in antiquity by Greek philosophers, through which this way of reasoning is exemplified. First a rule is provided: Every man is mortal. Next, a case is enunciated in particular: Aristotle is a man. Finally the feature which is proper to the individual is deduced: Aristotle is mortal. However, this is not the unique way of attaining the knowledge of something. If we alter the way these three elements are presented, it is possible to find two other ways of reaching a logical conclusion. These options can shown by graphs, where $\mathrm{R}$ is the rule, $\mathrm{C}$ the case and $\mathrm{f}$ the feature.

\section{DEDUCTION}

$$
\mathrm{R}+\mathrm{C} \rightarrow \mathrm{f}
$$

\section{ABDUCTION}

$$
\mathrm{R}+\mathrm{f} \rightarrow \mathrm{C}
$$

\section{INDUCTION}

$$
\mathrm{C}+\mathrm{f} \rightarrow \mathrm{R}
$$

If we frame the theme that gives cause to this work with an example in particular, it is feasible to reason how an inference arises by means of deduction. In effect, if we consider as valid the rule that says: every student who has an adequate knowledge of mathematics, and will respond the posed questions satisfactorily or will solve the problems adequately in an examination, and the case that the one who takes the exam is a student who has a fine knowledge and a firm management of the concepts about which he is being examined, then the feature that should be conveyed is: the student will respond satisfactorily to the questions he or she will be asked.

However, mathematical science has not considered abduction as valid for its reasoning, because it might lead to false conclusions arising from true premises or, inversely, true conclusions arising from false premises. Let us consider a non-scientific example so that we can validate this claim.

The following rule could be enunciated: every player on the English soccer team plays that sport well; next a case could be given in particular: Beckham is a player on the English soccer team; finally the feature that characterizes that athlete will be inferred: Beckham is a good soccer player. This is a process of valid reasoning which is framed in a deduction.

Now let us see what happens when abduction is made with the same example. The rule will be the same: every player on the English soccer team plays that sport well. We can present a case in particular: Ronaldo is not on the English soccer team, and then infer the feature that characterizes that athlete: Ronaldo is not a good soccer player, which is obviously false. As it can be seen, we have reached a false conclusion in spite of its true premises.

But it is possible to have the inverse situation, that is, to reach a true conclusion starting from a false premise. The rule is still the same: every player on the English soccer team plays that sport well. The (false) case is: Ronaldo is a player of the English soccer team. The inferred feature that characterizes that athlete in particular is then: Ronaldo is a good soccer player (true).

Now, the methods used to determine if the knowledge of students of mathematics is satisfactory are based-almost without exception—on abduction, in spite of the fact, as we have just seen, that there are risks that such reasoning does not lead to absolutely valid conclusions, and in spite of the practically nonexistent use of such a way of reasoning in the process of mathematical logic.

70 HeRnÁ n NOTTOLI- Methods for Evaluation in Mathematics for Architecture and Design 
The epistemologist Charles Pierce has studied the abductive process of inference, based on working with the following rule: every student who possesses the accurate mathematical knowledge will respond the posed questions satisfactorily, or will solve the problems in an examination accurately; he has considered the feature of the evaluated student: the student responds the posed question satisfactorily, and finally, he has reached by abduction the conclusion: the student possesses the necessary knowledge to pass the exam. But it may also happen that the student in question fulfills what the evaluation proposes - he or she responds to everything he is asked - and yet does not possess the sufficient knowledge to pass the exam, because has he or she neither acquired the basic concepts accurately nor does he know much of other topics of the syllabus. Or it may also happen that the student has followed the process of learning from memory, which has allowed him or her to transcribe previously read texts, without taking adequately internalizing the substance that each theme possesses, which will not enable the transfer of such knowledge to other disciplines or its application to problems the student might face in the future.

Therefore, the conclusion obtained through abduction is not highly reliable. However, this is the method used and recognized by every educational institution at present. And why is it reliable as an evaluation methodology? We shall now analyze the process known as induction, in order to arrive at a satisfactory explanation.

Let us analyse another approach to the same example. Let us modify the question to: should it be considered reliable to evaluate the knowledge of the student who responds to everything adequately, i.e., he has passed the exam? The adequate responses given by the student will be the observed case. The feature will be the following observation: the student has satisfactory knowledge of the subject. The conclusion will be the rule: every student that possesses accurate mathematical knowledge will respond the questions posed during an examination satisfactorily. In other words: an inference by induction.

The reiteration of these cases by induction constitutes the statistical justification that really supports the method of abduction as the way of evaluating, and makes predictable its reasonable degree of reliability.

Two particularities are stressed in these previous considerations: a priori in the case of abduction, and a posteriori in the case of induction.

Let us now analyse the mechanism to determine the effectiveness of the method for evaluating the knowledge of the students who are taking examinations.

In the course of mathematics of the School of Architecture of the University of Buenos Aires, a series of statistics has been worked out regarding the themes developed in the current syllabus of the subject, which also indicates the results that should obtained in the final examinations that the students have to take in order to pass the course.

It is important to point out that the mode of evaluation was first written and then oral, so that it could be possible to evaluate the knowledge of the students in depth; that the examination included open, closed and mixed type questions (an example of which will be developed in the Appendix to this paper); and that electronic support, such as calculators and graphic computers, was allowed but written texts on the discipline were not. The data which is relative to the themes developed in the course, subdivided according to each heading and percentage of time allotted, is shown in graph 1 . 

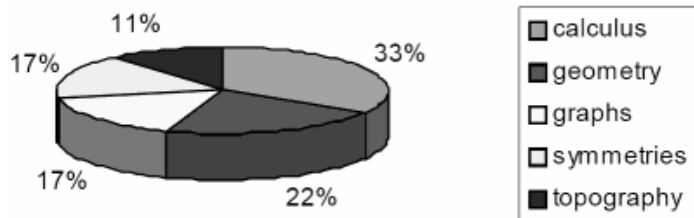

Graph 1

\section{Results of evaluations}

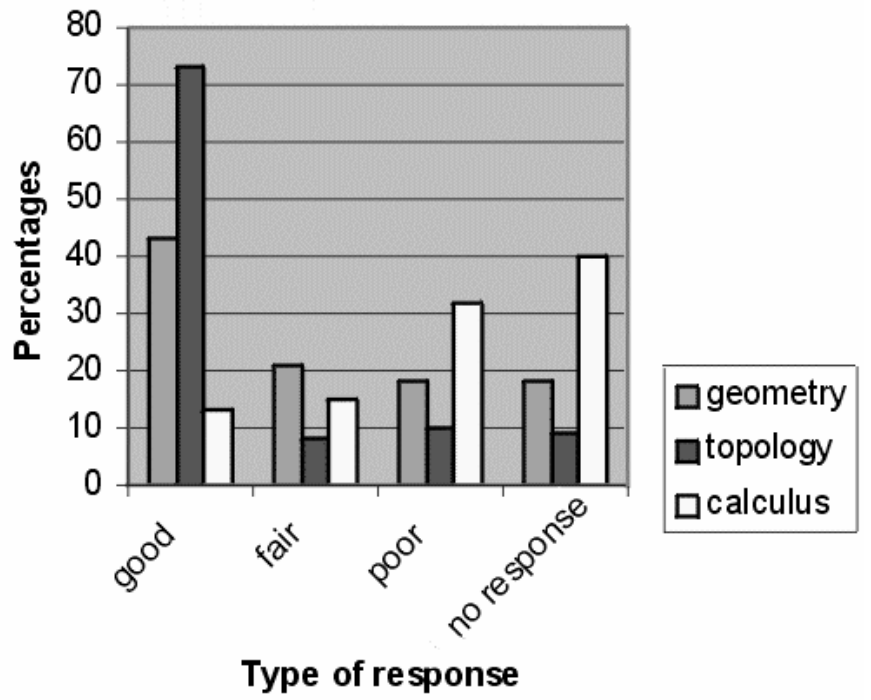

Graph 2

\section{General statistical data of the results of the evaluations}

The results obtained are summarized in a graph. We have grouped graphs and symmetry as topology; derivatives and integrals, and topography as calculus. So, it is possible to visualize the corresponding percentages for each heading, which show the diverse trends in the average evaluations being considered.

The data concerning the processed information are shown in graph $2 .^{1}$

The evaluated figures in percentiles in the graphs show that the students who study Architecture and Design have a higher affinity for themes that contain images (geometry, topography), or topological developments (graphs, symmetries), but not for calculus, which is a branch of mathematics that is always rejected by these students.

In order to optimize the procedure for determining if the knowledge of the students is adequate to pass mathematics, it must be emphasized that it is necessary to include diverse modes of 
questioning in the examinations. Our experience with having included problems containing open, closed and mixed-type questions has been highly positive.

Also, let us take into consideration that if the errors the students may make in an examination are not extremely serious, they should not be considered as such, if the students are warned of the errors committed and are able to solve them by themselves.

If we add the didactic and pedagogic bases that have been considered in the development of this issue, and the subsequent experiences in different courses-analyzed over time and comparatively in various schools of architecture and design-it could be concluded that there exist various possible ways to outline teaching strategies and to evaluate pupils preparing careers involving processes of designing. From all these ways, we think it is recommendable to choose as mechanisms of knowledge apprehension and methodologies of evaluation those that are more related to pupils who possess spatial intelligence. ${ }^{2}$

\section{Conclusions}

As a general conclusion, we believe it behooves us to incorporate as the main themes in the syllabi of mathematics of schools of architecture and design mathematical concepts presented through methodologies related to conceptual disciplines or reasoning-topological and graphicand to plan the evaluations with methodologies that are appropriate to those ways of thinking.

\section{Appendix}

In the proposed exercise the main point is to study an Eulerian path, that is, a tour in a connected graph (all its vertices are connected mutually by edges), in which all its edges are traveled passing by each one time. Figure 3 shows a graph in which it is possible to travel all its edges via the following Eulerian path: going from $\mathbf{a}$, to $\mathbf{b}$, to $\mathbf{c}$, then again to $\mathrm{a}$, then to $\mathrm{d}$ and finally to $\mathrm{c}$.

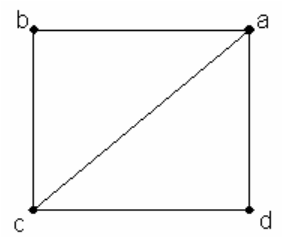

This type of path is of great usefulness in museums, exhibitions, etc., where the ideal paths are those in which it is possible to go through all the rooms or stands, without having to travel by the same place more than once. The example of the Barcelona Pavilion designed by Mies van der Rohe in 1929 is the case of a building conceived to exhibit paintings, sculptures, drawings, etc.; its spaces are continuous and "multiple use" and the exercise proposed in this article consists in finding in that building an Eulerian path (there could be more than one solution).

In general it is possible to determine a Eulerian path in diagrams of different configurations: ${ }^{3}$ 


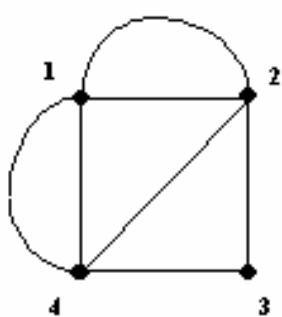

a)

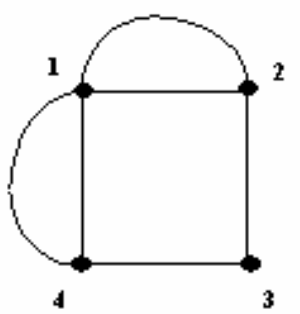

b)

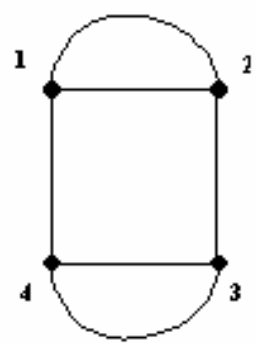

c)

a) Always, because all vertices are a point of contact for a number of edges.

b) Only starting from vertex 2 or 4, as an odd number of edges meet in vertices 2 and 4, and there are only two vertices with odd number of edges meeting in them.

c) It is never possible, because an odd number of edges meet in all vertices.

This type of topological and visual reasoning is, as we stated, very suitable for students of design schools, for it is easily applicable to this type of problem in design but not to others in which the fundamental component is calculation.

These types of exercises are useful from another point of view, for those teachers who usually use algebraic logical reasoning or deductions in which pure mathematical nomenclatures and codes are only inferred.

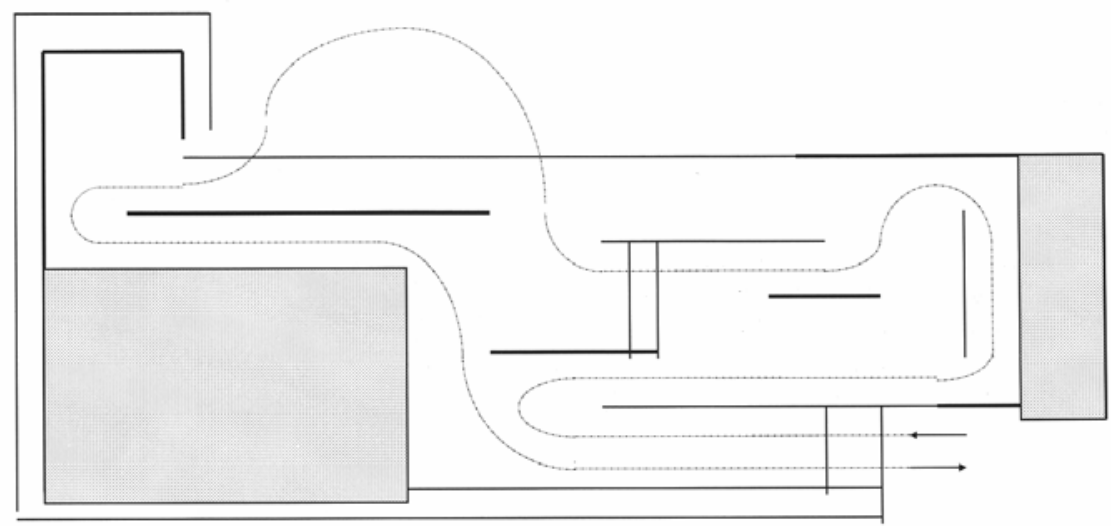

One possible Eulerian path that passes through every space in the Barcelona Pavillion 


\section{Notes}

1. The statistics have been worked out over a total of 2,892 exercises, solved by 536 students. The author sincerely thanks the collaboration given by the Faculty of his Chair of Mathematics II of the School of Architecture, Design and Urban Planning of the University of Buenos Aires, for the compilation and processing of these data.

2. H Gardner, Multiple intelligences: The theory in Practice, Perseus Book Group. 1993.

3. The name "eulerian" arises from the mathematician Leonard Euler (1707-1783). See V. Spinadel and H. Nottoli, Mathematical tools for Architecture and Design, Ed. FADU, 2005.

\section{About the author}

Hernán Nottoli possesses a wide and outstanding activity in teaching, and in apprenticeship fields in schools of design, in particular in subjects of technology. At present he is the Head of the Technological Area in the Faculty of Architecture, Design and Urban Planning in the University of Buenos Aires. He is also member of the Executive Council. He has developed a fruitful professional practice as designer in Argentina and also in Thun, Switzerland and in Johannesburg, South African Republic. He frequently lectures at universities all over the world.

He has published several books and articles in his country and abroad, and he has presented many papers in a great quantity of congresses and scientific meetings around the world. His curriculum appeared in the Dictionary Of International Biography of Cambridge, England. Currently, Architect Nottoli is Tenure Professor of Mathematics in the Faculty of Architecture of the University of Buenos Aires, and Main Researcher in the Secretariat of Science and Technology in the same School of Design. 